\title{
Modélisation physique en soufflerie et à l'extérieur du transport de neige par le vent
}

\author{
Florence Naaim-Bouvet et Mohamed Naaim \\ Division Nivologie/CEMAGREF
}

A priori le principe de la modélisation physique peut paraitre simple ; il s'agit de reproduire sur modèle réduit un épisode de transport de neige. Cependant se pose le problème de la validité des résultats (forme des accumulations, durée des tempêtes, ...). En effet le passage du modèle réduit à la réalité se fait par l'intermédiaire de critères de similitude; or l'ensemble de ces critères ne peuvent être satisfaits simultanément. Tous n'ont pas la même importance et de nombreux scientifiques (Imai, Gerdel, Strom, Odar, Anno, Kind, Iversen, Isyomov, Norem, ...) se sont intéressés à ce problème, mais étant donné la complexité des mécanismes, ces différents auteurs ne sont pas d'accord sur le groupe de critères le plus important à respecter. Reste donc à l'utilisateur à se forger sa propre opinion.

\section{Q MODÉLISATION PHYSIQUE EN SOUF- FLERIE}

\subsection{Critères de similitude}

\subsubsection{Rappels théoriques et notations}

Dans le cas d'une atmosphère stable, la vitesse moyenne du vent croit avec l'altitude suivant une loi logarithmique :

$$
U(h)-5,75 u_{*} \log \left(\frac{h}{z_{0}}\right) \text { avec } u_{*}-\sqrt{\frac{\Gamma_{p}}{\rho}}
$$

(avec $\Gamma_{p}$ contrainte exercée par le vent sur la surface du sol et $u_{*}$ vitesse de frottement).

Lorsque la vitesse du vent dépasse une valeur seuil, la force de frottement exercée sur les particules par le vent est responsable de la mise en mouvement de ces particules. La force de frottement seuil (et donc la vitesse de frottement seuil $u_{s_{t h}}$ ) dépend de la taille, de la forme, et du poids des particules. Le profil de vent devient alors le suivant (Bagnold [1]) :

$$
U(h)=5,75 u_{*} \log \left(\frac{h}{z_{0}^{\prime}}\right)+U_{t}
$$

\subsubsection{Modélisation physique par Kind [2] [3]}

Modélisation de la topographie : Les dimensions géométriques doivent toutes êtres réduites à l'échelle.
Modélisation de l'écoulement :

Similitude dynamique: Le flux doit être turbulent rugueux

$$
\frac{u_{s_{t h}}^{2}}{2 g v}>30
$$

Similitude cinématique : La rugosité (pendant la saltation) doit être à l'échelle géométrique

$$
k^{\prime} \propto \frac{u_{*}^{2}}{2 g}(\text { Owen }(1964)) \rightarrow\left(\frac{u_{*}^{2}}{2 H g}\right)_{m}=\left(\frac{u_{*}^{2}}{2 H g}\right)_{p}
$$

Modélisation de la saltation ;

trajectoire des particules:

$$
\left(\frac{U_{F}}{U}\right)_{m}=\left(\frac{U_{F}}{U}\right)_{p}
$$

$$
\left(\frac{U^{2}}{H g}\right)_{m}=\left(\frac{U^{2}}{H g}\right)_{p} \text { en considérant }\left(\frac{\sigma}{\sigma-\rho}\right)=1
$$

Débit des particules:

$$
\left(\frac{U_{* t h}}{U m}\right)=\left(\frac{u_{* t h}}{U}\right)_{p}
$$

Modélisation des dépôts :

$$
[\text { angle de repos }]_{m}=[\text { angle de repos }]_{p}
$$

Echelle des temps :

$$
\begin{gathered}
\left(\frac{t_{m}}{t_{p}}\right)_{\text {volumes accumulés }}=\frac{\rho_{p} / \rho_{p}}{\rho_{m} / \sigma_{m}} \times \frac{H_{m} / U_{m}}{H_{p} / U_{p}} \\
\left(\frac{t_{m}}{t_{p}}\right)_{\text {masses accumulées }}=\frac{H_{m} / U_{m}}{H_{p} / U_{p}}
\end{gathered}
$$

Remarque: Kind envisage l'abandon du nombre de Froude car le respect de ce dernier ne permet pas de diminuer de façon significative l'échelle.

\subsubsection{Modélisation physique par Iversen [4] [5] [6] [7] [8]}

Iversen propose la démarche suivante: une analyse dimensionnelle met en évidence les variables nécessaires à la description du phénomène de saltation ; ces paramètres 


\begin{tabular}{|c|c|}
\hline \multicolumn{2}{|r|}{ Notation } \\
\hline$L, I$ & : Longueur de référence $(\mathrm{m})$ \\
\hline k & : Rugosité équivalente $(\mathrm{m})$ \\
\hline$z_{0}$ & Rugosité aérodynamique $(\mathrm{m})$ \\
\hline$u$ & : Vitesse à la hauteur $h(\mathrm{~m} / \mathrm{s})$ \\
\hline$U_{0}$ & : Vitesse seuil à la hauteur $h(\mathrm{~m} / \mathrm{s})$ \\
\hline & : Vitesse seuil à la hauteur $z_{0}^{\prime}(\mathrm{m} / \mathrm{s})$ \\
\hline$U_{F}^{\prime}$ & : Vitesse de chute des particules $(\mathrm{m} / \mathrm{s})$ \\
\hline & : Masse volumique du fluide $\left(\mathrm{kg} / \mathrm{m}^{3}\right)$ \\
\hline$v$ & : Viscosité cinématique $\left(\mathrm{m}^{2} / \mathrm{s}\right)$ \\
\hline$A$ & $\begin{array}{l}\text { : Surface de la congère projetée suivant la } \\
\text { vertical }\left(\mathrm{m}^{2}\right)\end{array}$ \\
\hline$A e$ & $\begin{array}{l}\text { : Surface de la congère projetée suivant l'ho- } \\
\text { rizontale }\left(\mathrm{m}^{2}\right)\end{array}$ \\
\hline$t$ & : Temps de formation de la congère $(\mathrm{s})$ \\
\hline$Q_{s}$ & : Masse spécifique transportée $(\mathrm{kg} / \mathrm{ms})$ \\
\hline$n$ & $\begin{array}{l}\text { Coefficient d'interception des particules par } \\
\text { un piège }\end{array}$ \\
\hline$\lambda$ & : Longueur de la zone d'emprunt $(\mathrm{m})$ \\
\hline & : Modélisé (paramètre en soufflerie) \\
\hline$H, r$ & : Hauteur de référence $(\mathrm{m})$ \\
\hline & : Rugosité équivalente en saltation (m) \\
\hline & : Rugosité aérodynamique en saltation (m) \\
\hline$u_{*}$ & : Vitesse de frottement $(\mathrm{m} / \mathrm{s})$ \\
\hline$u_{*}$ th & Vitesse de frottement seuil $(\mathrm{m} / \mathrm{s})$ \\
\hline$D_{p}^{\prime \prime \prime}$ & : Diamètre de la particule $(\mathrm{m})$ \\
\hline & $\begin{array}{l}\text { : Masse volumique réelle des particules } \\
\left(\mathrm{kg} / \mathrm{m}^{3}\right)\end{array}$ \\
\hline$\gamma$ & $\begin{array}{l}\text { : Masse volumique apparente des particules } \\
\left(\mathrm{kg} / \mathrm{m}^{3}\right)\end{array}$ \\
\hline$g$ & : Accélération de la gravité $\left(\mathrm{m} / \mathrm{s}^{2}\right)$ \\
\hline & : Prototype (paramètre grandeur nature) \\
\hline
\end{tabular}

ne pouvant être satisfaits simultanément, il est possible de les combiner par des moyens théoriques et statistiques.

Modélisation de la topographie: L'échelle verticale peut différer de l'échelle horizontale.

Modélisation de l'écoulement :

Similitude cinématique: La rugosité (pendant la saltation) doit être à l'échelle géométrique.

$k^{\prime} \propto \frac{\rho u_{*}^{2}}{\sigma g}($ White (1976), Iversen (1979)) $\rightarrow$

$$
\rightarrow\left(\frac{\rho u_{*}^{2}}{\sigma H g}\right)_{m}=\left(\frac{\rho u_{*}^{2}}{\sigma g H}\right)_{p}
$$

c'est-à-dire

$$
\left(A_{1}^{2}\left(\frac{D_{p}}{H}\right)\left(\frac{u_{*}}{u_{* t h}}\right)^{2}\right)_{m}=\left(A_{1}^{2}\left(\frac{D_{p}}{H}\right)\left(\frac{u_{*}}{u_{* t h}}\right)^{2}\right)_{p}
$$

avec

$$
A_{1}=u_{* h} \sqrt{\frac{\rho}{\sigma g D_{p}}}
$$

Modélisation de la saltation :

Trajectoire des particules:

$$
\left(\frac{C_{D} \rho_{L}}{\sigma D_{p}}\right)_{m}=\left(\frac{C_{D} \rho_{L}}{\alpha D_{p}}\right)_{p}
$$

et

$$
\left(\frac{g L^{2}}{u_{*}^{2} H}\right)_{m}=\left(\frac{g L^{2}}{u_{*}^{2} H}\right)_{p}
$$

Prépondérance de la saltation :

$$
\frac{U_{F m}}{u_{*_{t h m} m}}>1
$$

Les paramètres (11) à (14) permettent de choisir les particules. Les paramètres (12) et (13) ne sont significatifs que lorsque de petites irrégularités affectent la forme des congères. Aussi Iversen veille-t-il simplement à respecter le critère (14) et l'ordre de grandeur de (11).

Modélisation des dépôts :

Taux de croissance adimensionnel:

Après une première proposition (1979) :

$$
\frac{d\left(A / L^{2}\right)}{d\left(\frac{u_{*} t}{L}\right)}=\left(\frac{\rho}{\sigma}\right)\left(\frac{u_{*}^{2}}{g H}\right)\left(1-\frac{u_{* t h}}{u_{*}}\right)
$$

Iversen envisage une forme plus générale dont il cherche une valeur analytique au cas par cas par une analyse statistique. En effet, il ne généralise pas la formule en considérant que le meilleur ajustement est une fonction de la géométrie :

$$
\begin{aligned}
& \frac{d\left(A / L^{2}\right)}{d\left(u_{*} t / L\right)}=\frac{\rho u_{*}^{2}}{\sigma g H}\left(1-\frac{u_{*} t h}{u_{*}}\right) \times \\
& \times \mathscr{F}\left(\frac{u(H) L}{v}, \frac{h}{H}, \frac{l}{L}, \frac{z_{0}}{H}, \frac{z_{0}^{\prime}}{H}, \frac{\rho}{\sigma}, \frac{U_{F}}{u_{*} h}, \frac{u_{*}^{2}}{g H}\right)
\end{aligned}
$$

Ainsi en considérant $\left(\Delta A / L^{2}\right)_{m}=\left(\Delta A / L^{2}\right)_{p}$, ceci lui a permis d'aboutir dans les deux cas particuliers qu'il a étudiés (croisement d'autoroute $((1980,1982) \rightarrow(16-a))$ et barrière de porosité $1 / 3((1984) \rightarrow(16-b))$ aux critères suivants :

$$
\begin{aligned}
& {\left[\frac{\left(A_{1}^{2}\left(\frac{D_{p}}{H}\right)\left(\frac{U}{U_{0}}\right)^{2}\right)^{3 / 7}}{\left.\left(\frac{U \Delta t}{L}\right)\left(\frac{\rho U^{2}}{\sigma g H}\right)\left(1-\frac{U_{0}}{U}\right)\right]_{m}}\right.} \\
& =\left[\frac{\left(A_{1}^{2}\left(\frac{D_{p}}{H}\right)\left(\frac{U}{U_{0}}\right)^{2}\right)^{3 / 7}}{\left(\frac{U \Delta t}{L}\right)\left(\frac{\rho U^{2}}{\sigma g H}\right)\left(1-\frac{U_{0}}{U}\right)}\right]_{p} \text { (16a) } \\
& {\left[\left(\frac{U \Delta t}{L}\right)\left(\frac{\rho U^{2}}{\sigma g H}\right)\left(1-\exp \left(-6,2\left(U / U_{0}-1\right)\right)\right)\right]_{m}=} \\
& {\left[\left(\frac{U \Delta t}{L}\right)\left(\frac{\rho U^{2}}{\sigma g H}\right)\left(1-\exp \left(-6,2\left(U / U_{0}-1\right)\right)\right)\right]_{p}}
\end{aligned}
$$

Afin de déterminer le rapport des vitesses et le rapport des temps, il est nécessaire d'effectuer une hypothèse supplémentaire. Dans un premier temps (1979), Iversen a proposé l'isochronie $\left((U \Delta t / L)_{m}=(U \Delta t / L)_{p}\right)$; mais comme le rapport de la vitesse de la particule sur la vitesse du vent 
n'est pas le même pour le modèle et le prototype, Iversen abandonne ce critère et propose l'utilisation du nombre de Froude densimétrique modifié :

$$
\begin{aligned}
{\left[\left(\frac{\rho}{\sigma}\right)\left(\frac{U^{2}}{g H}\right)\left(1-\frac{U_{0}}{U}\right)\right]_{m}=} \\
{\left[\left(\frac{\rho}{\sigma}\right)\left(\frac{U^{2}}{g H}\right)\left(1-\frac{U_{0}}{U}\right)\right]_{p} }
\end{aligned}
$$

\subsubsection{Modélisation physique par Anno [9] [10] [11]}

L'approche de Anno est essentiellement expérimentale ; il procède par éliminations successives des critères de moindre importance.

Modélisation de la topographie: Les dimensions géométriques doivent toutes être réduites à l'échelle et il est souhaitable que $D_{p} / D_{m}=L_{p} / L_{m}$.

Modélisation de l'écoulement : Le flux doit être turbulent rugueux.

Modélisation de la trajectoire et de l'éjection des particules :

$$
\left[\frac{u_{*}}{u_{* t h}}\right]_{m}=\left[\frac{u_{*}}{u_{* t h}}\right]_{p}
$$

Modélisation de la forme des congères :

$$
\text { [angle de repos }]_{m}=[\text { angle de repos }]_{p}
$$

Echelle des temps :

$$
\left[\frac{t Q n}{\gamma L^{2}}\right]_{p}=\left[\frac{t Q n}{\gamma L^{2}}\right]_{m}
$$

(Comparaison des volumes accumulés).

Remarque : les critères de similitude proposés par Anno s'appliquent aussi à la modélisation à l'extérieur.

\subsubsection{Modélisation physique par Tabler}

\section{[12] (modélisation à l'extérieur)}

Echelle géométrique : Toutes les grandeurs caractéristiques doivent être mises à l'échelle

$$
\frac{H_{m}}{H_{p}}=\frac{\left(z_{0}\right)_{m}}{\left(z_{0}\right)_{p}}
$$

Modélisation de l'écoulement :

$$
U(h)=5,75 u_{* t h} \log \left(\frac{h}{z_{0}}\right)
$$

Echelle dynamique: Nombre de Froude

$$
\frac{H_{m}}{H_{p}}=\frac{U_{m}^{2}}{U_{p}^{2}}
$$

Si l'on considère le respect des échelles géométriques (21) et dynamique (23), la condition à remplir devient :

$$
\frac{H_{m}}{H_{p}}=\frac{U_{m}^{2}}{U_{p}^{2}}=\frac{\left(u_{*}\right)_{m}^{2}}{\left(u_{*}\right)_{p}^{2}}=\frac{\left(z_{0}\right)_{m}}{\left(z_{0}\right)_{p}}
$$

Echelle des temps:

$\mathrm{Si}$ les densités de l'air, des particules et la masse volumique des congères sont les mêmes pour le modèle et le prototype, l'échelle des temps serait :

$$
\frac{t_{m}}{t_{p}}=\frac{H_{m} / U_{m}}{H_{p} / U_{m}}=\frac{L_{m} / u_{*_{m}}}{L / u_{*_{p}}}
$$

La masse volumique de la neige fraîche varie avec la température et l'humidité de l'air, la taille et la forme des flocons, la vitesse du vent et l'épaisseur de la congère. En fin de saison la masse volumique approche les $450 \mathrm{~kg} / \mathrm{m}^{3}$. Pour tenir compte de cela, on modifie l'équation (25) en :

$$
\frac{t_{m}^{\prime}}{t_{p}^{\prime}}=\frac{\gamma_{m}}{\gamma_{p}}\left(\frac{H_{m} / u_{*_{m}}}{H_{p} / u_{*_{p}}}\right)
$$

où $t^{\prime}$ est le temps d'obtention de la saturation.

\subsection{Comparaison des différents critères de similitude}

Face aux difficultés théoriques rencontrées, il reste donc à I'utilisateur à se forger sa propre opinion. C'est ce que nous avons fait en comparant des congères :

- obtenues en vraie grandeur [13] [14] campagne de mesures réalisées de 1982 à 1988 derrière des barrières à neige (porosité $50 \%$, garde au sol $0,2 \mathrm{H}$ ) sur le plateau de Besse-en-Chandesse (Massif central/France)) (fig. 2) - modélisées en soufflerie [15] (cf. schéma Grenoble/France) (figs. I et 2)

- modélisées à l'extérieur avec de la neige [16] (Chicoutimi/Québec) (fig. 4)

- modélisées à l'extérieur avec du sable [17] (Agadir/Maroc) (fig. 3).

\section{Géométrie générale :}

Si l'on compare globalement les profils obtenus en soufflerie et les mesures effectuées à Besse-en-Chandesse, deux différences importantes apparaissent. En soufflerie la congère au vent est quasi-inexistante et la congère sous le vent bien que semblable est cependant moins longue. Iversen [6] fait les mêmes constatations lorsqu'il compare ses simulations physiques avec les mesures in situ de Tabler [12].

Ceci pourrait s'expliquer par les deux phénomènes suivants :

- Transport par un vent d'une direction différente de celle du vent dominant.

- Chute de neige sans vent: d'une part on observe une modification des caractéristiques géométriques de la barrière (diminution de la garde au sol) et d'autre part on note un rehaussement de la congère de la hauteur $h$ de la chute de neige sans vent.

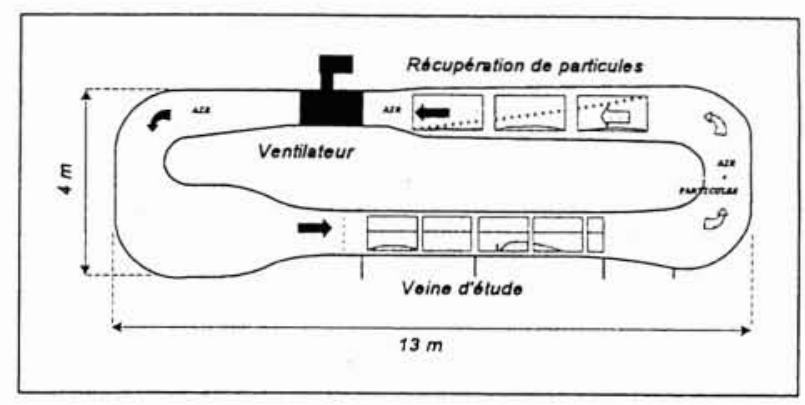

1. Soufflerie du CEMAGREF (Grenoble). 
MODÉLISATION PHYSIQUE DU TRANSPORT DE NEIGE

Tableau 1. - Grandeurs réelles modélisées

\begin{tabular}{|c|c|c|c|c|}
\hline \multirow{2}{*}{ Valeurs obtenues en sufflerie } & \multicolumn{4}{|c|}{ Valeurs réelles modélisées en utilisant les critères proposés par: } \\
\hline & Kind $^{1}$ & Iversen $^{2}$ & Tabler & Anno \\
\hline $\begin{array}{l}\text { Sciure D60/80 } \\
u_{*}=34 \mathrm{~cm} / \mathrm{s}\end{array}$ & & $\begin{array}{l}\text { Vitesse } \\
u_{*}=213 \mathrm{~cm} / \mathrm{s}\end{array}$ & $\begin{array}{c}\text { Vitesse } \\
u_{*}=271 \mathrm{~cm} / \mathrm{s}\end{array}$ & $\begin{array}{l}\text { Vitesse } \\
u_{*}=45 \mathrm{~cm} / \mathrm{s} \\
\text { Durée } \\
1141 \mathrm{~h}\end{array}$ \\
\hline $\begin{array}{l}\text { Verre } 90 / 150 \\
u_{*}=34 \mathrm{~cm} / \mathrm{s}\end{array}$ & $\begin{array}{l}\text { Vitesse } \\
\begin{array}{l}u_{*}=36 \mathrm{~cm} / \mathrm{s} \\
\text { Durée } \\
510 \mathrm{mn}\end{array}\end{array}$ & $\begin{array}{l}\text { Vitesse } \\
u_{*}=93 \mathrm{~cm} / \mathrm{s} \\
\text { Durée } \\
563 \mathrm{mn}\end{array}$ & $\begin{array}{c}\text { Vitesse } \\
u_{*}=128 \mathrm{~cm} / \mathrm{s} \\
\text { Durée } \\
541 \mathrm{mn}\end{array}$ & $\begin{array}{l}\text { Vitesse } \\
u_{*}=36 \mathrm{~cm} / \mathrm{s} \\
\text { Durée } \\
910 \mathrm{~h}\end{array}$ \\
\hline
\end{tabular}

1 Le nombre de Froude n'a pas été respecté.

2 Le critère d'Iversen a été utilisé au-delà de sa limite de validité $\left(A e H^{2}=8\right)$.

Les résultats obtenus dépendent des conditions initiales qui ont été prises identiques pour l'ensemble des critères $\left(z_{0 \text { neige }}=2 \mathrm{~mm}, z_{0 \text { soufflerie }}=0,04 \mathrm{~mm}, u_{\text {th neige }}=0,2 \mathrm{~cm} / \mathrm{s}, \sigma_{\text {neige }}=700 \mathrm{~kg} / \mathrm{m}^{3}\right)$.
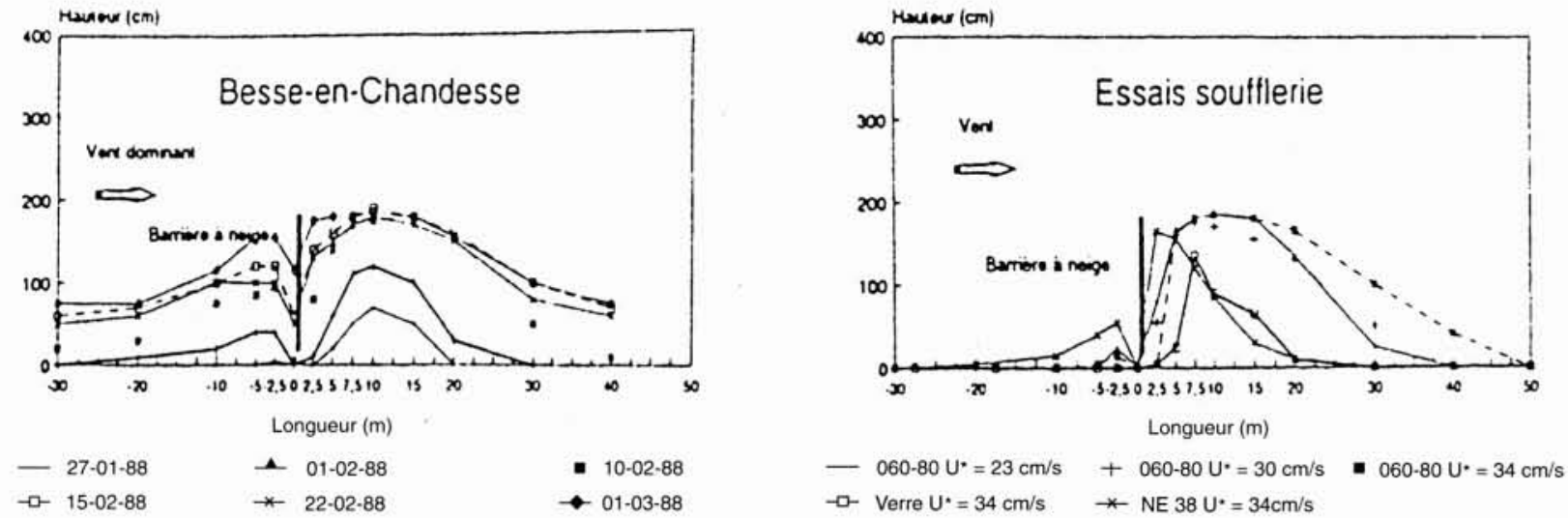

2. Comparaison modélisation en soufflerie et vraie grandeur (barrière à neige poreuse $50 \%$, garde au sol $0,2 \mathrm{H}$ ). N.B. Les congères générées par le verre et le sable NE38 n'ont pas atteint la saturation.

Cependant cette explication est infirmée par les expériences que nous avons réalisées en modélisation physique à l'extérieur avec de la neige (Chicoutimi/Québec) et avec du sable (Agadir/Maroc). Dans les deux cas la direction du vent a été constante et perpendiculaire à la barrière (barrière pleine avec garde au sol de hauteur $0,2 \mathrm{H}$ ).

Pour la modélisation à l'extérieur avec de la neige (fig. 4), la courte durée de l'expérience $(24 h)$ nous a permis de nous assurer que seul intervenait le phénomène de saltation. On ne peut donc imputer la différence de forme des congères au vent uniquement à des problèmes de chute de neige sans vent. Quant à la modélisation à l'extérieur avec du sable (fig. 3) elle nous permet de montrer que la mauvaise reproduction de la cohésion des particules ne permet pas d'expliquer ce phénomène.

\section{Vitesse :}

Les ordres de grandeur des vitesses sont corrects excepté dans le cas de Tabler et Iversen (Utilisation du nombre de Froude) où la vitesse semble surestimée. Une expérience réalisée en soufflerie (fig. 6) montre que ce dernier critère,
Barnère porosité $0 \% \mathrm{H}=16.25 \mathrm{~cm}$ Garde au sol $0.2 \mathrm{H}$

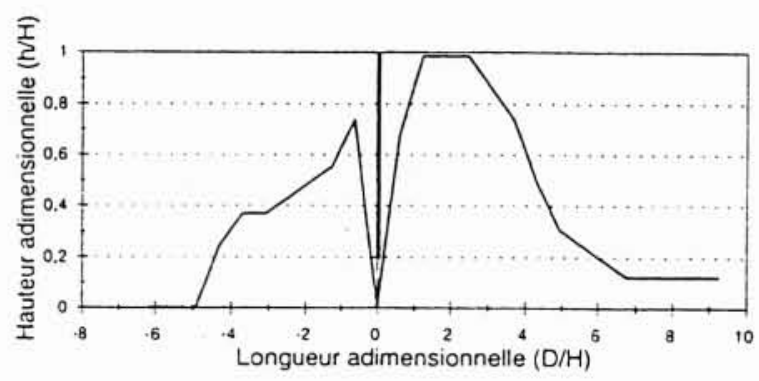

Modélisation à l'extérieur (sable)

3. Modélisation à l'extérieur avec du sable (barrière pleine avec garde au sol de $0,2 \mathrm{H}$ ). 
lorsqu'il est utilisé seul (sans considération vis-à-vis des autres critères de similitude issus de la modélisation des trajectoires des particules) n'est pas valable. Par ailleurs, la modélisation de rugosité en l'absence de saltation (fig. 7) n'entraîne que peu de modification sur la forme des congères sous le vent.

Ceci ne signifie pas pour autant que les expériences réalisées par Tabler (modélisation physique à l'extérieur avec de la neige sur un lac gelé) ne soient pas valides, bien au contraire. On peut appliquer aux résultats obtenus par Tabler les critères de similitude de Anno.

Pour les autres critères, la bonne adéquation entre grandeur réelle et grandeur modélisée s'explique d'une part par la faible sensibilité (qui dépend il est vrai de la forme de l'obstacle) de la forme des congères à la vitesse (fig. 5) et d'autre part par l'imprécision des données grandeur réelle (une congère réelle ne se forme pas à vitesse constante; la vitesse de formation de la congère est donc estimée).

Cependant notre préférence ira au critère proposé par Anno, car c'est l'un des rares expérimentateurs qui montre par des essais la validité ou du moins la prédominance de son critère. Un point cependant est à préciser : la vitesse de frottement seuil apparaissant dans le critère (18) est une vitesse de frottement seuil de dépôt et non d'arrachement.

Barrière porosité $0 \% \mathrm{H}=11,25 \mathrm{~cm}$ Garde au sol $0,2 \mathrm{H}$

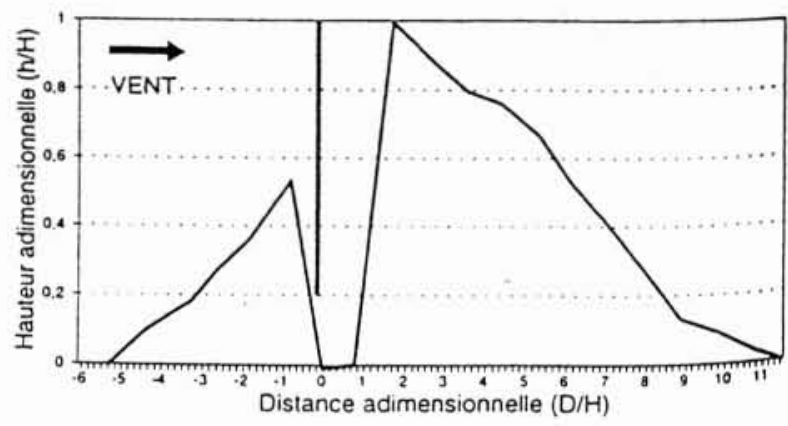

—Modélisation á l'extérieur (neige)

4. Modélisation à l'extérieur avec de la neige (barrière pleine avec garde au sol de $0,2 \mathrm{H}$ ).

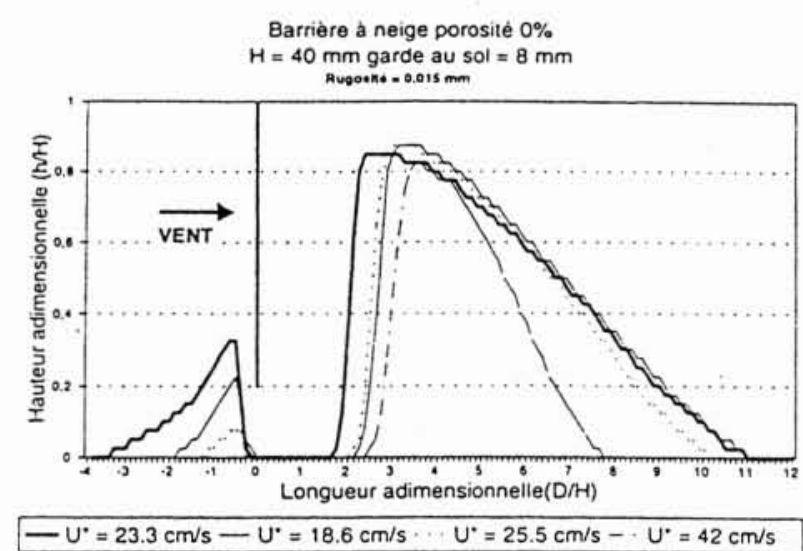

5. Modélisation en soufflerie avec de la sciure (barrière pleine avec garde au sol de $0,2 \mathrm{H}$ ).
Si à aucun moment Anno ne précise ce point dans son article, la justification théorique qu'il développe ne peut s'appliquer que dans ces conditions. Par ailleurs le dispositif expérimental utilisé conduit à la mesure de la vitesse de frottement seuil de dépôt et non à la vitesse de frottement seuil d'arrachement.

\section{Durée de tempête :}

En ce qui concerne l'estimation des durées réelles de tempête, les divergences sont importantes. L'échelle des temps proposée par Kind et Tabler ne peut représenter les durées de tempête car $U_{p}$ et $U_{m}$ sont les vitesses de vent ou de particules en mouvement et non les vitesses de dépôts ou d'érosion.

Bien que présentés différemment, les raisonnements basés sur la comparaison des volumes accumulés par Iversen et Anno sont semblables. L'estimation de $n$, coefficient d'interception, dont on ne connaît pas a priori la valeur, et qui dépend de l'obstacle et de l'échelle utilisée, rend difficile l'utilisation du critère proposé par Anno. Dans la formulation d'Iversen, $n$ apparaît implicitement dans la fonction $\mathscr{F}$ dont on détermine expérimentalement la forme

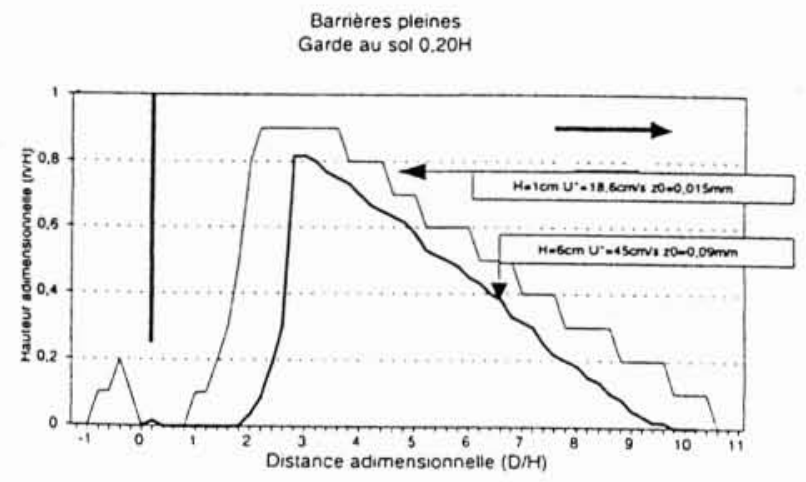

6. Le seul critère de Froude n'est pas adapté : les courbes ne se superposent pas.

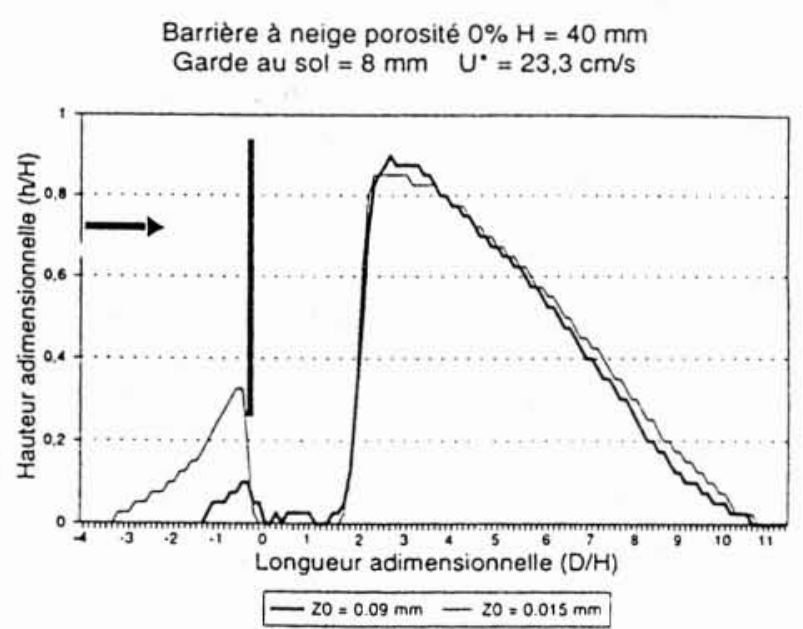

7. Faible sensibilité de la forme des congères à la rugosité en l'absence de saltation. 


$$
\begin{aligned}
\frac{d\left(A / L^{2}\right)}{d\left(u_{*} t / L\right)}=\frac{\rho u_{*}^{2}}{\sigma g H} & \left(1-\frac{u_{*_{t} h}}{u_{*}}\right) \times \\
& \quad \times \mathscr{F}\left(\frac{u(H) L}{v}, \frac{h}{H}, \frac{l}{L}, \frac{z_{0}}{H}, \frac{z_{0}^{\prime}}{H}, \frac{\rho}{\sigma}, \frac{U_{F}}{u_{*} h}, \frac{u_{*}^{2}}{g H}\right)
\end{aligned}
$$

Cependant dans les expériences d'Iversen, la longueur de la zone de reprise est fixée à $1,4 \mathrm{~m}$. Il est donc légitime de se demander si pour cette longueur la saturation en particules est atteinte. En effet dans le cas contraire, il faut introduire dans la fonction $\mathscr{F}$ une nouvelle variable, qui est la longueur de la zone d'emprunt dont dépend le débit $Q_{s}$. Ceci a été confirmé par les expériences que nous avons réalisées en soufflerie (fig. 8 , fig. 9).

On constate donc que lorsque les profils de concentration diffèrent, toutes choses égales par ailleurs, il n'y a plus superposition des courbes $\rightarrow$

$$
\begin{aligned}
\frac{d\left(A / L^{2}\right)}{d\left(u_{*} t / L\right)}= & \frac{\rho u_{*}^{2}}{\sigma g H}\left(1-\frac{u_{* t h}}{u_{*}}\right) \times \\
& \times \mathscr{F}^{\prime}\left(\lambda, \frac{u(H) L}{v}, \frac{h}{H}, \frac{l}{L}, \frac{z_{0}}{H}, \frac{z_{0}^{\prime}}{H}, \frac{\rho}{\sigma}, \frac{U_{F}}{u_{*}, h}, \frac{u_{*}^{2}}{g H}\right)
\end{aligned}
$$

avec $\lambda$ longueur de la zone d'emprunt

Il est difficile de savoir a posteriori si, lors de ses expériences, Iversen obtenait en soufflerie la saturation en particules. On ne connaît que peu de résultats expérimentaux sur les problèmes d'obtention de la saturation en particules, d'autant plus que le sujet est complexe en soufflerie, puisqu'il y a développement en parallèle de la couche limite. De plus dans notre cas $u_{*}$ augmente le long de la veine d'étude puisque la section de la soufflerie est constante, ce qui ne permet pas de compenser le développement de la couche limite. Si l'on se réfère à Takeuchi [20], il semblerait que la longueur de la zone d'emprunt nécessaire à la saturation soit plus importante. Ceci semble confirmé lorsque l'on compare les résultats obtenus par Iversen en soufflerie et par Tabler en modélisation extérieure.

Iversen [8] compare donc les résultats obtenus par luimême en soufflerie [6] et par Tabler [11] en modélisation extérieure avec de la neige sur les mêmes barrières (po-

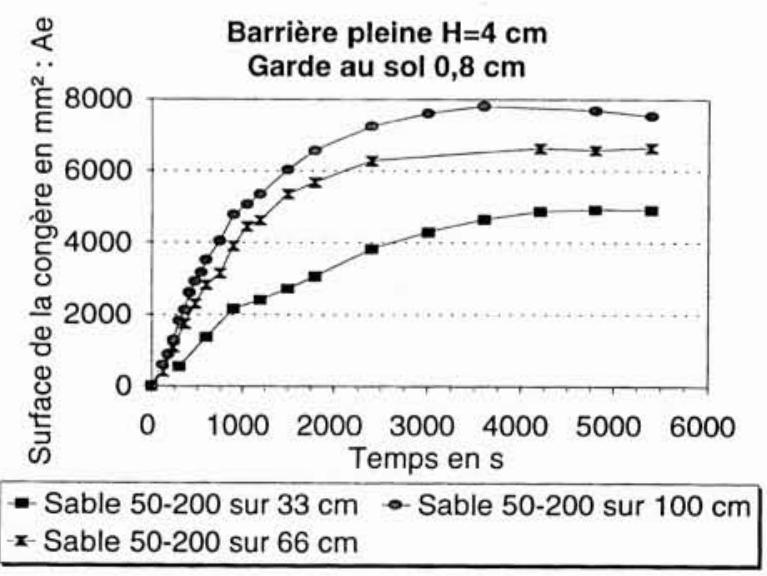

8. Influence de la longueur de la zone reprise. rosité de $50 \%$ avec une garde au sol de $0,1 \mathrm{H}$ ). Dans les deux cas il utilise le paramètre dérivé du débit des particules.

Et alors qu'en soufflerie il obtient la relation :

$$
\begin{array}{r}
\left(\frac{\Delta A}{L^{2}}=4,98(10)^{-4}\left(\frac{U_{\infty} \Delta t}{L}\right)\left(\frac{\rho U_{\infty}^{2}}{\sigma g H}\right)(1-\exp \right. \\
\left.\left.\left(-6,2\left(U / U_{t}-1\right)\right)\right)\right)
\end{array}
$$

en modélisation extérieur il arrive à :

$$
\begin{array}{r}
\left(\frac{\Delta A}{L^{2}}=9,89(10)^{-4}\left(\frac{U_{\infty} \Delta t}{L}\right)\left(\frac{\rho U_{\infty}^{2}}{\sigma g H}\right)(1-\exp \right. \\
\left.\left.\left(-6,2\left(U / U_{t}-1\right)\right)\right)\right)
\end{array}
$$

Iversen n'explique pas cette différence (il existe un facteur 2 entre les deux formulations) qui peut être simplement due au fait que dans le cas d'Iversen, et d'une façon plus générale en soufflerie, la saturation n'est pas atteinte.

L'utilisation du critère d'Iversen, lorsque la saturation en particules n'est pas atteinte conduit donc à une surestimation de la durée réelle des temps de formation des congères.

Par ailleurs l'expérience ( $f i g .8$ ) que nous avons réalisée en soufflerie permet de mettre en évidence un point important. Le comportement asymptotique des courbes semble différer, ce qui signifie donc que le profil de concentration en particules influe sur la forme des congères. La vitesse de référence pour les trois expériences est prise à l'entrée de la veine avant la zone de reprise des particules. Ainsi la modification du profil de vitesse du fait de la présence des particules [1], [21] en plus ou moins grande quantité (transfert de quantité de mouvement) est suffisante pour avoir un effet sur la forme des congères.

Par contre, lorsque la saturation est atteinte, nous avons pu constater la validité du critère proposé par Iversen. Nous avons réalisé une modélisation à l'extérieur avec du sable (zone d'emprunt de $200 \mathrm{~m}$ ). Le seul paramètre que nous pouvions faire varier était la hauteur des barrières. En

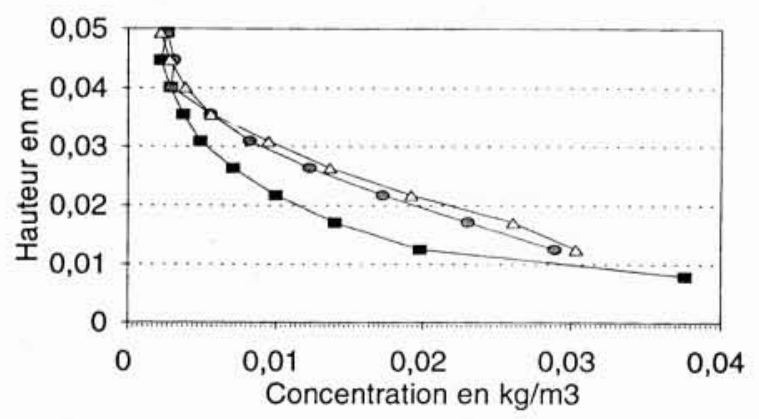

- Sable 50-200 sur $33 \mathrm{~cm}$ a Sable $50-200$ sur $100 \mathrm{~cm}$ $\triangle$ Sable $50-200$ sur $200 \mathrm{~cm}$

9. Profils de concentration de correspondant (obtenus par traitement d'images [18], [19]). 
Barrière pleine $\mathrm{H}=4 \mathrm{~cm}$

Garde au sol $0,8 \mathrm{~cm}$

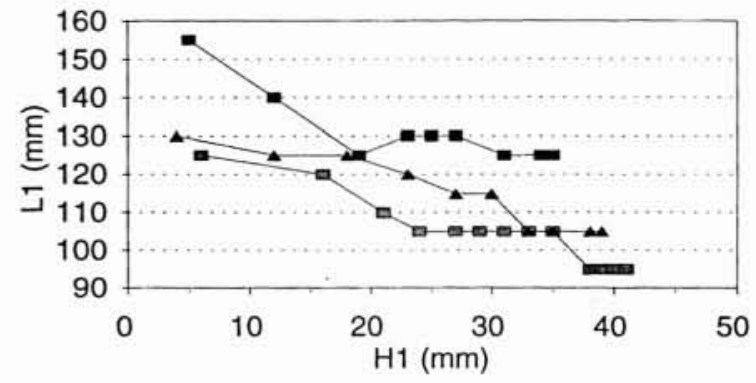

- Sable 50-200 sur $33 \mathrm{~cm}$ - Sable $50-200$ sur $100 \mathrm{~cm}$ - Sable 50-200 sur $66 \mathrm{~cm}$

10. Influence de la concentration en particules sur l'évolution de la forme de la congère pour une même vitesse de référence.

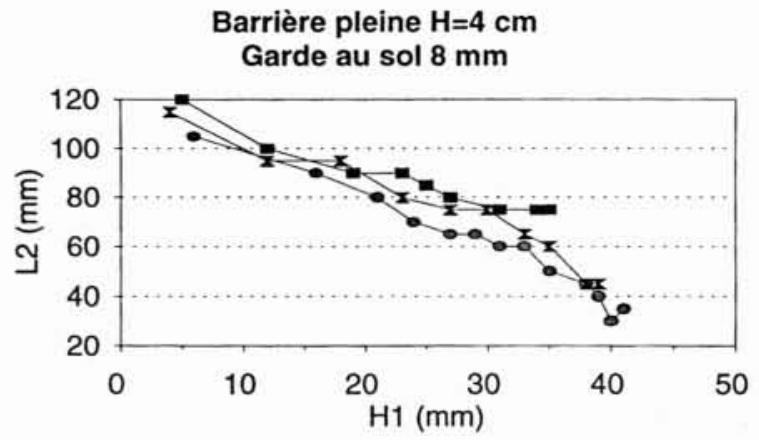

- Sable 50-200 sur $33 \mathrm{~cm}$ - Sable 50-200 sur $100 \mathrm{~cm}$ - Sable $50-200$ sur $66 \mathrm{~cm}$

11. Influence de la concentration en particules sur l'évolution de la forme de la congère pour une même vitesse de référence.

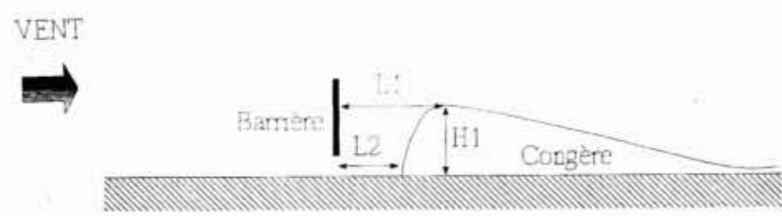

utilisant le critère (16a), qui prend en compte la modélisation de la rugosité pendant la saltation, conjugué au critère (18), (critère de vitesse proposé par Anno), on remarque une superposition des courbes, ce qui valide donc bien le critère proposé par Iversen (fig. 12).

\section{II $\square$ CONCLUSIONS}

Pour ce qui est de la forme des dépôts, la modélisation physique en soufflerie ou à l'extérieur donne de bons

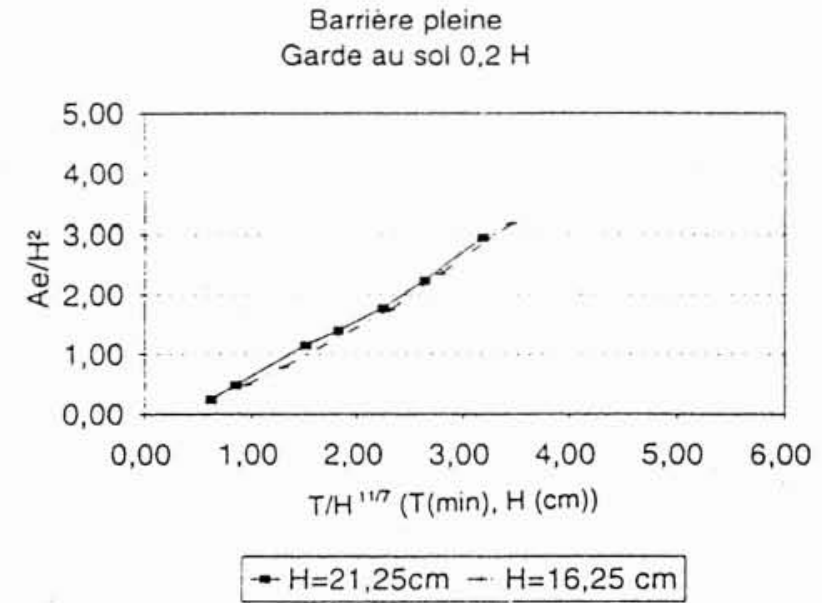

12. Validation du critère (16a) pour des profils de concentration à saturation.

résultats. Si les différences observées ont une importance du point de vue théorique, elles n'en ont pas du point de vue de l'ingénierie. Pour la gestion d'un réseau routier, il est capital de connaître le temps de formation d'une congère; or en l'état actuel des connaissances, la modélisation physique en soufflerie ne permet pas de répondre à cette demande. On s'oriente donc actuellement vers la modélisation numérique de la formation des congères.

\section{Remerciements}

Nos remerciements vont à $\mathrm{F}$. Sarret et $\mathrm{A}$. Bouddour pour la réalisation des expérimentations de modélisation à l'extérieur ainsi qu'à l'Université de Chicoutimi/Québec ( $G$. Lemieux / G. Vachon / laboratoire de télédétection) et l'Université d'Agadir/Maroc ( $M$. Ikenne/département de géologie) pour leur soutien logistique.

\section{Bibliographie}

[1] BAGNOLD R.A. (1941), - The physics of blownsands and desert dunes. London, Methuen.

[2] KIND J.D. (1975). - A critical examination of the requirements for model simulation of wind induced erosion/deposition phenomena such as a snowdrifting. Atmospheric Environment, Vol. 10, p. 219-227.

[3] KIND J.D. (1985). - Snowdrifting : a review of modelling. methods. Department of mechanical and aeronautical Engineering, Carleton University, Ottawa, Ontario, K1S5B6.

[4] IVERSEN J.D. (1979), - Drifting snow similitude - Drift deposit rate correlation. Unpublished (paper presented at Fifth International Conference on Wind Enginnering. Colorado, Fort Collins).

[5] IVERSEN J.D. (1980). - Driftingsnow similitude - Transport rate and roughness modeling. Journal of Glaciology, Vol. 26 No. 94.

[6] IVERSEN J.D. (1981), - Comparison of wind-tunnel model and full-scale snow fence drift. Journal of Wind Engineering and Industrial Aerodynamics, 8, pp. 231-249. 
[7] IVERSEN J.D. (1982), - Small-scale modeling of drifting snow phenomena. Wind tunnel Modeling for Civil Engineering Applications, Cambridge University Press, p. 522545 .

[8] IVERSEN J.D. (1984). - Comparaison of snowdrift modeling criteria : commentary on \& Application of Anno's modeling conditions to outdoor modeling of snowdrifts ». Cold Regions Science and Technology, 9, p. 259-265.

[9] ANNO Y. (1984). - Requirements for modeling a snowdrift. Cold Regions Science and Technologh, 8, p. 241-252.

[10] ANNO Y. (1984). - Froude number paradoxes in the modeling of a snowdrift. Cold regions science and technology, pp. 179-181.

[11] ANNO Y. (1984). - Applications of Anno's modeling conditions to outdoor modeling of snowdrifts. Cold Regions Science and Technology, 9, pp. 179-181.

[12] TABLER R.D. (1980). - Self similarity of wind profiles in blowing snow allows outdoor modeling. Journal of Glaciology, vol. 26, pp. 421-434.

[13] CEMAGREF/CETE (1982 à 1988), - Essais comparatifs de barrières à neige. Rapports annuels.

[14] NaAim F. et BRUgnot G. (1992). - Transport de la neige par le vent: connaissances de base et recommandations. CEMAGREF de Grenoble ISBN 2-85362-312-2.
[15] NAAIM F. (1990). - Transport de la neige par le vent: Modélisation physique en soufflerie diphasique. Mémoire de DEA MMGE Université Joseph Fourrier Grenoble 1.

[16] SARRET F. (1993). - Modélisation des congères à l'extérieur et en soufflerie. CEMAGREF/Université du Québec.

[17] Bouddour A. (1994). - Modélisation physique du transport de sable par le vent à l'extérieur (plage d'Agadir). CEMAGREF/Université d'Agadir.

[18] Martinez M. et NaAim M. (1992). - Application $d u$ traitement d'images à la mesure du transport de la diffusion des particules dans un écoulement chargé. Congrès Société Hydrotechnique de France.

[19] Martinez M. et NaAim M. (1993), - Détermination expérimentale et numérique du profil vertical de concentration dans un écoulement chargé. $11^{\mathrm{e}}$ congrès français de mécanique/Lille-Villeneuve d'Asq.

[20] TAKEUCHI M. (1980), - Vertical profile and horizontal increase of drift snow transport. Journal of Glaciology, vol. $26, \mathrm{n}^{\circ} 94$, pp. 481-492.

[21] KIKUCHI T. (1981), - A wind tunnel study of the aerodynamic roughness associated with drifting snow. Cold Regions Science and Technology, 5, pp. 107-118. 